\title{
Asymptotic behavior of the Verblunsky coefficients for the OPUC with a varying weight
}

Cite as: J. Math. Phys. 53, 043510 (2012); https://doi.org/10.1063/1.4705276

Submitted: 03 July 2010 . Accepted: 04 April 2012 . Published Online: 25 April 2012

M. Poplavskyi

Don't let your writing keep you from getting published!
AIP Author Services

Learn more today! 


\title{
Asymptotic behavior of the Verblunsky coefficients for the OPUC with a varying weight
}

\author{
M. Poplavskyia) \\ Mathematical Division, B. Verkin Institute for Low Temperature Physics, National Academy \\ of Sciences of Ukraine, 47 Lenin Ave., Kharkiv 61103, Ukraine
}

(Received 3 July 2010; accepted 4 April 2012; published online 25 April 2012)

\begin{abstract}
We present an asymptotic analysis of the Verblunsky coefficients for the polynomials orthogonal on the unit circle with the varying weight $e^{-n V(\cos x)}$, assuming that the potential $V$ has four bounded derivatives on $[-1,1]$ and the equilibrium measure has a one interval support. We obtain the asymptotics as a solution of the system of "string" equations. (c) 2012 American Institute of Physics. [http://dx.doi.org/10.1063/1.4705276]
\end{abstract}

\section{INTRODUCTION}

In this paper we consider a system of polynomials orthogonal on the unit circle (OPUC) with a varying weight. The system can be obtained from $\left\{e^{i k \lambda}\right\}_{k=0}^{\infty}$ if we use the Gram-Shmidt procedure in $L^{(n)}:=L_{2}\left([-\pi, \pi], e^{-n V(\cos \lambda)}\right)$ with the inner product

$$
\langle f, g\rangle_{n}=\int_{-\pi}^{\pi} f(x) \overline{g(x)} e^{-n V(\cos x)} d x .
$$

Then for any $n$ we get the system of trigonometric polynomials

$$
P_{k}^{(n)}(\lambda)=\sum_{l=0}^{k} c_{k, l}^{(n)} e^{i l \lambda}
$$

which are orthonormal in $L^{(n)}$. One can see from the Szegö's condition (see e.g. Ref. 17) that the system $\left\{P_{k}^{(n)}(\lambda)\right\}_{k=0}^{\infty}$ is not complete in $L^{(n)}$. To construct the complete system one should include also polynomials with respect to $e^{-i \lambda}$. Hence following Ref. 3 we consider reversed polynomials

$$
Q_{k}^{(n)}(\lambda)=e^{i k \lambda} P_{k}^{(n)}(-\lambda)=\sum_{l=0}^{k} c_{k, l}^{(n)} e^{i(k-l) \lambda}
$$

and define the set of functions $\left\{\chi_{k}^{(n)}(\lambda)\right\}_{k=0}^{\infty}$

$$
\begin{aligned}
\chi_{2 k}^{(n)}(\lambda) & =e^{-i k \lambda} Q_{2 k}^{(n)}(\lambda), \\
\chi_{2 k+1}^{(n)}(\lambda) & =e^{-i k \lambda} P_{2 k+1}^{(n)}(\lambda) .
\end{aligned}
$$

It is easy to check (see, e.g., Ref. 3) that the system $\left\{\chi_{k}^{(n)}(\lambda)\right\}_{k=0}^{\infty}$ is an orthonormal basis in $L^{(n)}$. Moreover, it was proven in Ref. 3 that the functions $\chi_{k}^{(n)}$ satisfy the five term recurrent relations

$$
\begin{aligned}
e^{i \lambda} \chi_{2 k-1}^{(n)}(\lambda)= & -\alpha_{2 k}^{(n)} \rho_{2 k-1}^{(n)} \chi_{2 k-2}^{(n)}(\lambda)-\alpha_{2 k}^{(n)} \alpha_{2 k-1}^{(n)} \chi_{2 k-1}^{(n)}(\lambda) \\
& -\alpha_{2 k+1}^{(n)} \rho_{2 k}^{(n)} \chi_{2 k}^{(n)}(\lambda)+\rho_{2 k}^{(n)} \rho_{2 k+1}^{(n)} \chi_{2 k+1}^{(n)}(\lambda),
\end{aligned}
$$

\footnotetext{
a)Electronic mail: poplavskiymihail@rambler.ru.
} 


$$
\begin{aligned}
e^{i \lambda} \chi_{2 k}^{(n)}(\lambda)= & \rho_{2 k}^{(n)} \rho_{2 k-1}^{(n)} \chi_{2 k-2}^{(n)}(\lambda)+\alpha_{2 k-1}^{(n)} \rho_{2 k}^{(n)} \chi_{2 k-1}^{(n)}(\lambda) \\
& -\alpha_{2 k+1}^{(n)} \alpha_{2 k}^{(n)} \chi_{2 k}^{(n)}(\lambda)+\alpha_{2 k}^{(n)} \rho_{2 k+1}^{(n)} \chi_{2 k+1}^{(n)}(\lambda),
\end{aligned}
$$

where $\alpha_{k}^{(n)}=c_{k, 0}^{(n)} / c_{k, k}^{(n)}$ and $\rho_{k}^{(n)}=c_{k-1, k-1}^{(n)} / c_{k, k}^{(n)}$ are called the Verblunsky coefficients of the system $\left\{\chi_{k}^{(n)}(\lambda)\right\}_{k=0}^{\infty}$. They satisfy the relations

$$
\left(\rho_{k}^{(n)}\right)^{2}+\left(\alpha_{k}^{(n)}\right)^{2}=1
$$

Hence the matrix of the operator $C$ of multiplication by $e^{i \lambda}$ in the basis $\left\{\chi_{k}^{(n)}(\lambda)\right\}_{k=0}^{\infty}$ has the form (see Ref. 3)

$$
C^{(n)}=\left(\begin{array}{ccccccc}
-\alpha_{1}^{(n)} & \rho_{1}^{(n)} & 0 & 0 & 0 & 0 & \ldots \\
-\rho_{1}^{(n)} \alpha_{2}^{(n)} & -\alpha_{1}^{(n)} \alpha_{2}^{(n)} & -\rho_{2}^{(n)} \alpha_{3}^{(n)} & \rho_{2}^{(n)} \rho_{3}^{(n)} & 0 & 0 & \ldots \\
\rho_{1}^{(n)} \rho_{2}^{(n)} & \alpha_{1}^{(n)} \rho_{2}^{(n)} & -\alpha_{2}^{(n)} \alpha_{3}^{(n)} & \alpha_{2}^{(n)} \rho_{3}^{(n)} & 0 & 0 & \ldots \\
0 & 0 & -\rho_{3}^{(n)} \alpha_{4}^{(n)} & -\alpha_{3}^{(n)} \alpha_{4}^{(n)} & -\rho_{4}^{(n)} \alpha_{5}^{(n)} & \rho_{4}^{(n)} \rho_{5}^{(n)} & \ldots \\
0 & 0 & \rho_{3}^{(n)} \rho_{4}^{(n)} & \alpha_{3}^{(n)} \rho_{4}^{(n)} & -\alpha_{4}^{(n)} \alpha_{5}^{(n)} & \alpha_{4}^{(n)} \rho_{5}^{(n)} & \ldots \\
0 & 0 & 0 & \ddots & \ddots & \ddots & \ddots
\end{array}\right) .
$$

The main goal of the paper is to find the first two terms of the asymptotic expansion of $\alpha_{n+k}^{(n)}$ for $k=o(n), n \rightarrow \infty$. The asymptotics of polynomials orthogonal with respect to a varying weight plays an important role in many problems of modern mathematics, e.g., approximation theory, combinatorics, theory of random matrices, etc. The polynomials orthogonal with respect to a varying weight on the real line are now well studied, due to the remarkable paper (Ref. 4), where it was shown that for real analytic $V(\lambda)$ satisfying also some growth conditions as $|\lambda| \rightarrow \infty$, the asymptotics of polynomials $P_{n+k}^{(n)}(\lambda)(k=o(n), n$ $\rightarrow \infty$ ) orthogonal on the real line (OPRL) with the weight $e^{-n V(\lambda)}$, is expressed via $\theta$ functions, which can be constructed in terms of the so-called equilibrium measure $\mu(d \lambda)$ $=\rho(\lambda) d \lambda$, that is obtained as a solution of a classical problem of the potential theory with the potential $V$. These results were generalized on the case of $V$ possessing only few derivatives in the work (Ref. 10). The complete asymptotic expansion of the Jacobi matrix coefficients in the case of real analytic $V$ and one or two interval equilibrium density $\rho$ was constructed in Ref. 1 . These results were used to prove universality of local bulk and edge regimes of the hermitian matrix models with the potential $V$ (see also Refs. 8,13, and 14). The case of OPUC, with varying weight till now is not understood so well as the case of classical OP. It is believed that similarly to the real line case, the asymptotics is closely connected with the behavior of the corresponding equilibrium measure, which is the minimizer of the functional

$$
\mathcal{E}[m]=\int_{-\pi}^{\pi} V(\cos \lambda) m(d \lambda)-\int_{-\pi}^{\pi} \log \left|e^{i \lambda}-e^{i \mu}\right| m(d \lambda) m(d \mu),
$$

in the class of unit measures on the interval $[-\pi, \pi]$ (see Ref. 16 for existence and properties of the solution). It is well known, in particular, that for smooth $V^{\prime}$ the equilibrium measure has a density $\rho$ which is uniquely defined by the condition that the function

$$
u(\lambda)=V(\cos \lambda)-2 \int_{\sigma} \log \left|e^{i \lambda}-e^{i \mu}\right| \rho(\mu) d \mu,
$$

takes its minimum value if $\lambda \in \sigma=\operatorname{supp} \rho$. From this condition in the case of differentiable $V$ one can obtain the following integral equation for the equilibrium density $\rho$

$$
(V(\cos \lambda))^{\prime}=v \cdot p \cdot \int_{-\pi}^{\pi} \cot \frac{\lambda-\mu}{2} \rho(\mu) d \mu, \quad \text { for } \lambda \in \sigma .
$$


The asymptotics of OPUC with varying weight was constructed in Ref. 9 for the case when the support $\sigma$ of $\rho$ is the whole circle. It was proven that in this case $P_{n}^{(n)}(\lambda) \sim C_{V} e^{i n \lambda}$ and $\alpha_{n}^{(n)}=\bar{o}(1)$. In the present paper we find the first two terms of the asymptotic expansion of $\alpha_{n+k}^{(n)}$ in the case of one-interval support $\sigma$ of the equilibrium density $\rho$. Our main conditions are

Condition C1: The support $\sigma$ of the equilibrium measure is a single subinterval of the interval $[-\pi, \pi]$, i.e.

$$
\sigma=[-\theta, \theta] \text {, with } \theta<\pi
$$

Condition C2: The equilibrium density $\rho$ has no zeros in $(-\theta, \theta)$ and

$$
\rho(\lambda) \sim C|\lambda \mp \theta|^{1 / 2}, \text { for } \lambda \rightarrow \pm \theta,
$$

and the function $u(\lambda)$ of (1.5) attains its minimum if and only if $\lambda$ belongs to $\sigma$.

Condition C3: $V(\cos \lambda)$ possesses 4 bounded derivatives on $\sigma_{\epsilon}=[-\theta-\varepsilon, \theta+\varepsilon]$.

Remark 1: In fact there is one more possibility to have one-interval $\sigma$. Another case is a some left symmetric arc of the circle, i.e., $[\pi-\theta, \pi+\theta]$. In this case we replace $V(\cos x)$ by $V(\cos (\pi-x))$. After this replacement we obtain the support from the condition $C 1$. The scalar product $\left\langle e^{i k x}, e^{i m x}\right\rangle_{n}$ will change on factor $(-1)^{k-m}$. Therefore, one can see from the Gram-Shmidt algorithm, that coefficients $\alpha_{k}^{(n)}$ will change on factor $(-1)^{k}$.

The following simple representation of $\rho$ plays an important role in our asymptotic analysis

Proposition 2: Under conditions C1-C3

$$
\rho(\lambda)=\frac{1}{4 \pi^{2}} \chi(\lambda) P(\lambda) \mathbf{1}_{\sigma}
$$

where

$$
\chi(\lambda)=\sqrt{|\cos \lambda-\cos \theta|}, \quad P(\lambda)=\int_{-\theta}^{\theta} \frac{(V(\cos \mu))^{\prime}-(V(\cos \lambda))^{\prime}}{\sin (\mu-\lambda) / 2} \frac{d \mu}{\chi(\mu)} .
$$

The proof is very simple but for the reader's convenience we give it in the beginning of Sec. III. It is evident that conditions $\mathrm{C} 1-\mathrm{C} 3$ imply that $P(\lambda)$ does not have roots on interval $[-\theta, \theta]$.

Remark 3: If the support of the equilibrium measure is the whole interval $[-\pi, \pi]$, then Eq. (1.6) can be solved using the Hilbert operator.

$$
\rho(\mu)=\frac{1}{2 \pi}+\frac{1}{4 \pi^{2}} \int_{-\pi}^{\pi} \cot \frac{\lambda-\mu}{2}\left((V(\cos \lambda))^{\prime}-(V(\cos \mu))^{\prime}\right) d \lambda .
$$

The main result of the paper is the following theorem:

Theorem 4: Consider the system of orthogonal polynomials and the Verblunsky coefficients defined in (1.2) and (1.3). Let potential $V$ satisfy conditions C1 - C3 above. Then there exists $\varepsilon_{1}>0$ such that for any $|m|<\varepsilon_{1} n$

$$
\alpha_{n+m}^{(n)}=(-1)^{n+m} s \cos \left(\frac{\theta}{2}+x_{n+m}^{(n)}\right)
$$

where $s=1$ or $s=-1$ and

$$
x_{n+m}=\frac{\pi \sqrt{2}}{P(\theta) \sin \theta / 2} \frac{m}{n}+\underline{O}\left(\log ^{11} n\left(n^{-4 / 3}+\frac{m^{2}}{n^{2}}\right)\right),
$$

with P defined in Eq. (1.10). 
Remark 5: The sign of s cannot be explicitly found and may be different for different values of $n$. As we are going to use this result in future for the proving of universality conjecture on the edge of spectrum, we expect that the sign of s will not affect the final result (for the example see Ref. 13).

The proof of Theorem 4 is based on the relation, which is an analog of the string equation for OP on the real line

Proposition 6: Let $\chi_{k}^{(n)}$ be the orthogonal functions defined in Eq. (1.1). Then for any $k$

$$
\int_{-\pi}^{\pi}(\sin \lambda) V^{\prime}(\cos \lambda) \chi_{k}^{(n)}(\lambda) \overline{\chi_{k-1}^{(n)}(\lambda)} e^{-n V(\cos \lambda)} d \lambda=i(-1)^{k-1} \frac{k}{n} \frac{\alpha_{k}^{(n)}}{\rho_{k}^{(n)}}
$$

The result was obtained in Ref. 5 but in some different form, therefore we give its proof in Sec. III. We use Eq. (1.13) as a system of nonlinear equations for $\alpha_{k}^{(n)}$ and solve it by the perturbation theory method. To construct a zero order solution of the system (1.13), following the method of Refs. 1 and 13, we use the link of the OPUC with the unitary matrix models, which eigenvalue distribution has the form

$$
p_{n}\left(\lambda_{1}, \ldots, \lambda_{n}\right)=\frac{1}{Z_{n}} \prod_{1 \leq j<k \leq n}\left|e^{i \lambda_{j}}-e^{i \lambda_{k}}\right|^{2} \exp \left\{-n \sum_{j=1}^{n} V\left(\cos \lambda_{j}\right)\right\} .
$$

It is known (see Ref. 11) that all marginal densities of Eq. (1.14) can be expressed using the orthogonal functions

$$
\psi_{k}^{(n)}(\lambda)=P_{k}^{(n)}(\lambda) e^{-n V(\cos \lambda) / 2}
$$

and their reproducing kernel

$$
K_{k, n}(\lambda, \mu)=\sum_{l=0}^{k-1} \psi_{l}^{(n)}(\lambda) \overline{\psi_{l}^{(n)}(\mu)}
$$

These ensembles first appeared in physics, but later their relationship to the pure mathematical problems was discovered (see, e.g., Ref. 2).

The paper is organized as follows. In Sec. II, we prove the main Theorem 4 using some technical results. We will prove these results in Sec. III.

\section{PROOF OF BASIC RESULTS}

The method of the proof is a version of the one used in Ref. 13 An important part of the proof is a zero order approximation for the Verblunsky coefficients $\alpha_{n+k}^{(n)}$.

Theorem 7: Under conditions of Theorem 4 we have

$$
\alpha_{n+k}^{(n)}=(-1)^{n+k} s \cos \frac{\theta}{2}+O\left(n^{-1 / 4} \log ^{1 / 2} n+\left(\frac{|k|}{n}\right)^{1 / 2}\right) .
$$

Proof of Theorem 7. The main idea of the proof is to derive an equation with a functional parameter $\phi$ for functions $\psi_{k}^{(n)}$ of Eq. (1.15) from the determinant formulas. Then, choosing appropriate parameter $\phi$, we obtain the equation for the Verblunsky coefficients. Define a new eigenvalue distribution

$$
p_{k, n}\left(\lambda_{1}, \ldots, \lambda_{k}\right)=Q_{k, n}^{-1} \prod_{1 \leq j<m \leq k}\left|e^{i \lambda_{j}}-e^{i \lambda_{m}}\right|^{2} \exp \left\{-n \sum_{l=1}^{k} V\left(\cos \lambda_{l}\right)\right\},
$$


which differs from Eq. (1.14) by the number of variables ( $k$ instead of $n)$. Let

$$
\begin{aligned}
\tilde{\rho}_{k, n}(\lambda) & =\int p_{k, n}\left(\lambda, \lambda_{2}, \ldots, \lambda_{k}\right) d \lambda_{2} \ldots d \lambda_{k}, \\
\widetilde{\rho}_{k, n}(\lambda, \mu) & =\int p_{k, n}\left(\lambda, \mu, \lambda_{3}, \ldots, \lambda_{k}\right) d \lambda_{3} \ldots d \lambda_{k},
\end{aligned}
$$

be the first and the second marginal densities of Eq. (2.2). By the standard argument (see Ref. 11) we have

$$
\begin{gathered}
\tilde{\rho}_{k, n}(\lambda)=k^{-1} K_{k, n}(\lambda, \lambda), \\
\widetilde{\rho}_{k, n}(\lambda, \mu)=\frac{1}{k(k-1)}\left[K_{k, n}(\lambda, \lambda) K_{k, n}(\mu, \mu)-\left|K_{k, n}(\lambda, \mu)\right|^{2}\right],
\end{gathered}
$$

where $K_{k, n}$ defined in Eq. (1.16). We will use also the notation

$$
\rho_{k, n}(\lambda) \equiv \frac{1}{n} K_{k, n}(\lambda, \lambda)=\frac{k}{n} \widetilde{\rho}_{k, n}(\lambda)
$$

Let $\phi$ be any $2 \pi$-periodic twice differentiable function. Then integrating by parts and using that $\frac{d}{d \lambda}\left|e^{i \lambda}-e^{i \mu}\right|^{2}=\cot \frac{\lambda-\mu}{2}\left|e^{i \lambda}-e^{i \mu}\right|^{2}$, we get

$$
\begin{aligned}
\int_{-\pi}^{\pi}(V(\cos \lambda))^{\prime} \tilde{\rho}_{k+n, n}(\lambda) \phi(\lambda) d \lambda=n^{-1} & \int_{-\pi}^{\pi} \widetilde{\rho}_{k+n, n}(\lambda) \phi^{\prime}(\lambda) d \lambda \\
& +\frac{n+k-1}{n} \int_{-\pi}^{\pi} \widetilde{\rho}_{k+n, n}(\lambda, \mu) \frac{\phi(\lambda)}{\tan (\lambda-\mu) / 2} d \lambda d \mu .
\end{aligned}
$$

Combining the above relation with the symmetry property $\widetilde{\rho}_{k+n, n}(\lambda, \mu)=\widetilde{\rho}_{k+n, n}(\mu, \lambda)$, we obtain

$$
\begin{aligned}
\int_{-\pi}^{\pi}(V(\cos \lambda))^{\prime} \tilde{\rho}_{k+n, n}(\lambda) \phi(\lambda) d \lambda=n^{-1} & \int_{-\pi}^{\pi} \tilde{\rho}_{k+n, n}(\lambda) \phi^{\prime}(\lambda) d \lambda \\
& +\frac{n+k-1}{2 n} \int_{-\pi}^{\pi} \tilde{\rho}_{k+n, n}(\lambda, \mu) \frac{\phi(\lambda)-\phi(\mu)}{\tan (\lambda-\mu) / 2} d \lambda d \mu .
\end{aligned}
$$

Using Eqs. (2.4) and (2.5), and the equality

$$
\int_{-\pi}^{\pi}\left|K_{k+n, n}(\lambda, \mu)\right|^{2} d \mu=K_{k+n, n}(\lambda, \lambda),
$$

we obtain from the above

$$
\begin{aligned}
\int_{-\pi}^{\pi} \frac{\phi(\lambda)-\phi(\mu)}{2 \tan (\lambda-\mu) / 2} \rho_{k+n, n}(\lambda) \rho_{k+n, n}(\mu) d \lambda d \mu \\
-\int_{-\pi}^{\pi}(V(\cos \lambda))^{\prime} \rho_{k+n, n}(\lambda) \phi(\lambda) d \lambda+\delta_{k, n}[\phi]=0,
\end{aligned}
$$

where

$$
\delta_{k, n}[\phi]:=\frac{1}{2 n^{2}} \int_{-\pi}^{\pi}\left[\phi^{\prime}(\lambda)+\phi^{\prime}(\lambda)-\frac{\phi(\lambda)-\phi(\mu)}{\tan (\lambda-\mu) / 2}\right]\left|K_{k+n, n}(\lambda, \mu)\right|^{2} d \lambda d \mu .
$$

We replace in Eq. (2.6) $k$ by $k-1$, subtract the new relation from Eq. (2.6), and multiply the result by $n$. Then we have

$$
\begin{aligned}
\int_{-\pi}^{\pi} \frac{\phi(\lambda)-\phi(\mu)}{\tan (\lambda-\mu) / 2} \rho(\mu)\left|\psi_{k+n}^{(n)}(\lambda)\right|^{2} d \lambda d \mu-\int_{-\pi}^{\pi}(V(\cos \lambda))^{\prime} \phi(\lambda)\left|\psi_{k+n}^{(n)}(\lambda)\right|^{2} d \lambda \\
+\delta_{k, n}^{(1)}[\phi]+\delta_{k, n}^{(2)}[\phi]=0
\end{aligned}
$$


where

$$
\begin{aligned}
\delta_{k, n}^{(1)}[\phi]:=\frac{1}{n} \int_{-\pi}^{\pi}\left[\phi^{\prime}(\lambda)+\phi^{\prime}(\mu)-\frac{\phi(\lambda)-\phi(\mu)}{\tan (\lambda-\mu) / 2}\right] & \cdot \Re\left\{K_{k+n-1, n}(\lambda, \mu) \overline{\psi_{k+n}^{(n)}(\lambda)} \psi_{k+n}^{(n)}(\mu)\right\} d \lambda d \mu, \\
\delta_{k, n}^{(2)}[\phi]:=\int_{-\pi}^{\pi} \frac{\phi(\lambda)-\phi(\mu)}{\tan (\lambda-\mu) / 2}\left(\rho_{n+k-1, n}(\mu)-\rho(\mu)\right)\left|\psi_{k+n}^{(n)}(\lambda)\right|^{2} d \lambda d \mu & +\frac{1}{n} \int \phi^{\prime}(\lambda)\left|\psi_{k+n}^{(n)}(\lambda)\right|^{2} d \lambda .
\end{aligned}
$$

To estimate $\delta_{k, n}^{(1)}[\phi]$ we note that

$$
\left|\phi^{\prime}(\lambda)+\phi^{\prime}(\mu)-\frac{\phi(\lambda)-\phi(\mu)}{\tan (\lambda-\mu) / 2}\right| \leq C\left|e^{i \lambda}-e^{i \mu}\right|\left(\left\|\phi^{\prime}\right\|_{\infty}+\left\|\phi^{\prime \prime}\right\|_{\infty}\right) .
$$

and use also the bound (see e.g., Ref. 15)

$$
\int\left|K_{k+n-1, n}(\lambda, \mu)\right|^{2}\left|e^{i \lambda}-e^{i \mu}\right|^{2} d \lambda d \mu \leq C .
$$

Combining it with the Schwartz inequality, we have

$$
\begin{aligned}
&\left|\delta_{k, n}^{(1)}[\phi]\right| \leq \frac{C}{n}\left(\left\|\phi^{\prime}\right\|_{\infty}+\left\|\phi^{\prime \prime}\right\|_{\infty}\right)\left(\int\left|K_{k+n-1, n}(\lambda, \mu)\right|^{2}\left|e^{i \lambda}-e^{i \mu}\right|^{2} d \lambda d \mu\right)^{1 / 2} \\
& \cdot \int\left|\psi_{k+n}^{(n)}(\lambda) \psi_{k+n}^{(n)}(\mu)\right|^{2} d \lambda d \mu \leq \frac{C}{n}\left(\left\|\phi^{\prime}\right\|_{\infty}+\left\|\phi^{\prime \prime}\right\|_{\infty}\right) .
\end{aligned}
$$

For $\delta_{k, n}^{(2)}[\phi]$ we use the following result of Ref. 7. Let $\rho_{n}:=p_{1}^{(n)}$ be the first marginal density of Eq. (1.14). Then $\rho_{n}$ converges weakly to the equilibrium density $\rho$ and for any $\phi \in H^{1}(-\pi, \pi)$

$$
\left|\int \phi(\lambda) \rho_{n}(\lambda) d \lambda-\int \phi(\lambda) \rho(\lambda) d \lambda\right| \leq C\|\phi\|_{2}^{1 / 2}\left\|\phi^{\prime}\right\|_{2}^{1 / 2} n^{-1 / 2} \ln ^{1 / 2} n,
$$

where $\|\cdot\|_{2}$ denotes $L_{2}$ norm on $[-\pi, \pi]$. Since the function $\frac{\phi(\lambda)-\phi(\mu)}{\tan (\lambda-\mu) / 2}$ has a bounded derivative with respect to $\mu$, we obtain

$$
\begin{aligned}
\left|\delta_{k, n}^{(2)}[\phi]\right| & \leq\left|\delta_{1, n}^{(2)}[\phi]\right|+C \frac{|k|}{n}\left\|\phi^{\prime}\right\|_{\infty} \\
\leq & =\left(\left\|\phi^{\prime}\right\|_{\infty}^{1 / 2}\left\|\phi^{\prime \prime}\right\|_{\infty}^{1 / 2} n^{-1 / 2} \log ^{1 / 2} n+\frac{|k|}{n}\left\|\phi^{\prime}\right\|_{\infty}\right) .
\end{aligned}
$$

We split integral in Eq. (2.8) into two parts. Using Eq. (1.6) for $\lambda \in \sigma$, we obtain

$$
\begin{aligned}
v \cdot p \cdot \int_{\sigma} d \lambda \int_{\sigma} d \mu \phi(\mu) \cot \frac{\lambda-\mu}{2} \rho(\mu)\left|\psi_{k+n}^{(n)}(\lambda)\right|^{2} & \\
- & -\int_{\sigma^{c}} d \lambda\left|\psi_{k+n}^{(n)}(\lambda)\right|^{2}\left(\int_{\sigma} d \mu \rho(\mu) \frac{\phi(\lambda)-\phi(\mu)}{\tan (\lambda-\mu) / 2}-\phi(\lambda)(V(\cos \lambda))^{\prime}\right) \\
& =\delta_{k, n}^{(1)}[\phi]+\delta_{k, n}^{(2)}[\phi] .
\end{aligned}
$$

Equation (1.6) is valid only for $\lambda \in \sigma$. Below we need to extend this relation on some neighborhood of $\sigma$. Using Eq. (1.9), we obtain the following representation. 
Lemma 8: Under the conditions of Theorem 4, for $\varepsilon$ defined in $C 3$ and $\lambda \in \sigma_{\varepsilon}=[-\theta-\varepsilon, \theta+\varepsilon], V$ can be represented in the form

$$
\begin{aligned}
(V(\cos \lambda))^{\prime}-\frac{1}{4 \pi^{2}} \int_{-\theta}^{\theta} \cot \frac{\lambda-\mu}{2} & P(\mu) \chi(\mu) d \mu \\
= & \frac{\operatorname{sign} \lambda}{2 \pi}\left(P(\lambda) \chi(\lambda)+O\left(\chi^{2}(\lambda)\right)\right) \mathbf{1}_{\sigma_{\varepsilon} \backslash \sigma}, \text { for } \lambda \rightarrow \pm \theta .
\end{aligned}
$$

Proofs of this lemma and other auxiliary results can be found in Sec. III of the paper. For $\lambda \in \sigma_{\varepsilon}^{c}$ we use the exponential bounds for the functions $\psi_{k}^{(n)}$. These bounds can be proved analogously to the corresponding result of Ref. 14 .

Proposition 9: Let potential $V$ satisfy conditions $C 1-C 3$. Than there exist constants $C, C_{1}>0$ and $\varepsilon_{1}>0$ such that for any integer $k$ satisfying inequality $|k|<\varepsilon_{1} n$ we have

$$
\int_{\sigma_{\varepsilon_{n, k}}}\left|\psi_{k+n}^{(n)}(\lambda)\right|^{2} d \lambda \leq e^{-C n^{1 / 2} \log n}, \quad \varepsilon_{n, k}=C_{1}\left(n^{-1 / 2} \log n+|k| / n\right) .
$$

Combining Eqs. (2.9), (2.11), (2.12), and (2.13) with Eq. (2.14), we obtain finally the integral equation

$$
\begin{aligned}
& v . p . \int_{\sigma_{\varepsilon_{n, k}}} d \lambda \int_{-\theta}^{\theta} d \mu \phi(\mu) \cot \frac{\lambda-\mu}{2} \rho(\mu)\left|\psi_{k+n}^{(n)}(\lambda)\right|^{2} \\
&+\frac{1}{2 \pi} \int_{\sigma_{\varepsilon_{n, k}} \backslash \sigma} \operatorname{sign} \lambda \phi(\lambda) P(\lambda) \chi(\lambda)\left|\psi_{k+n}^{(n)}(\lambda)\right|^{2} d \lambda \\
&=\left(\|\phi\|_{\infty}+\left\|\phi^{\prime}\right\|_{\infty}+\left\|\phi^{\prime \prime}\right\|_{\infty}\right) O\left(\varepsilon_{n, k}\right)
\end{aligned}
$$

with $\varepsilon_{n, k}$ of Eq. (2.14).

Remark 10: If the support of the equilibrium measure is the whole interval $[-\pi, \pi]$, then we can obtain in a similar way the integral equation for the equilibrium density

$$
v . p . \int_{-\pi}^{\pi} \phi(\mu) \cot \frac{\lambda-\mu}{2} \rho(\mu)\left|\psi_{k+n}^{(n)}(\lambda)\right|^{2} d \lambda d \mu=\left(\left\|\phi^{\prime}\right\|_{\infty}+\left\|\phi^{\prime \prime}\right\|_{\infty}\right) O\left(n^{-1 / 2} \log n+\frac{|k|}{n}\right) \text {. }
$$

It is easy to see that $P(\lambda)$ of Eq. (1.10) is a twice differentiable strictly positive function on the interval $[-\theta, \theta]$. Taking in Eq. (2.15)

$$
\phi(\mu)=P^{-1}(\mu) \cos \frac{\mu}{2} \cot \frac{z-\mu}{2},
$$

we obtain

$$
\begin{aligned}
& \int_{\sigma_{\varepsilon_{n}}}\left|\psi_{k+n}^{(n)}(\lambda)\right|^{2} f(z, \lambda, \theta) d \lambda \\
& \quad+2 \pi \int_{\sigma_{\varepsilon_{n}} \backslash \sigma} \operatorname{sign} \lambda \cot \frac{z-\lambda}{2} \cos \frac{\lambda}{2} \chi(\lambda)\left|\psi_{k+n}^{(n)}(\lambda)\right|^{2} d \lambda=O\left(\varepsilon_{n, k}|\operatorname{dist}(z, \sigma)|^{-3}\right),
\end{aligned}
$$

where

$$
f(z, \lambda, \theta)=v \cdot p \cdot \int_{-\theta}^{\theta} \cot \frac{\lambda-\mu}{2} \cot \frac{z-\mu}{2} \chi(\mu) \cos \frac{\mu}{2} d \mu .
$$


Computing $f(z, \lambda, \theta)$ we obtain

$$
\begin{gathered}
f(z, \lambda, \theta)=v \cdot p \cdot \int_{-\theta}^{\theta} \frac{\sin \mu+\sin \lambda}{\cos \mu-\cos \lambda} \frac{\sin \mu+\sin z}{\cos \mu-\cos z} \chi(\mu) \cos \frac{\mu}{2} d \mu \\
\quad=v \cdot p \cdot \int_{-\theta}^{\theta} \frac{\sin ^{2} \mu+\sin \lambda \sin z}{(\cos \mu-\cos \lambda)(\cos \mu-\cos z)} \chi(\mu) \cos \frac{\mu}{2} d \mu \\
=v \cdot p \cdot \int_{-\theta}^{\theta}\left[-1+\cot \frac{z-\lambda}{2}\left(\frac{\sin \lambda}{\cos \mu-\cos \lambda}-\frac{\sin z}{\cos \mu-\cos z}\right)\right] \chi(\mu) \cos \frac{\mu}{2} d \mu \\
=I_{1}+\cot \frac{z-\lambda}{2}\left(\sin \lambda I_{2}(\lambda)-\sin z I_{2}(z)\right),
\end{gathered}
$$

where

$$
I_{1}=-\int_{-\theta}^{\theta} \chi(\mu) \cos \frac{\mu}{2} d \mu=-\pi \sqrt{2} \sin ^{2} \frac{\theta}{2}, \quad I_{2}(z)=v \cdot p \cdot \int_{-\theta}^{\theta} \frac{\chi(\mu)}{\cos \mu-\cos z} \cos \frac{\mu}{2} d \mu .
$$

To compute $I_{2}(z)$ we use well known relations (see Ref. 12)

$$
S q_{a}(z):=\frac{1}{\pi} v \cdot p \cdot \int_{-a}^{a} \frac{d t}{(z-t) \sqrt{a^{2}-t^{2}}}=\left\{\begin{array}{cc}
1 / \sqrt{z^{2}-a^{2}}, & \text { for } z \notin[-a, a], \\
0, & z \in(-a, a),
\end{array}\right.
$$

where $1 / \sqrt{z^{2}-a^{2}}$ is defined by the asymptotic $\sim 1 / z, z \rightarrow \infty$. Then we have for $z \neq \pm \theta$

$$
I_{2}(z)=\sqrt{2} \int_{-1}^{1} \frac{\sqrt{1-t^{2}} d t}{\sin ^{2}(z / 2) / \sin ^{2}(\theta / 2)-t^{2}}=\sqrt{2} \pi\left(1-\frac{\sqrt{\sin ^{2} z / 2-\sin ^{2} \theta / 2}}{\sin z / 2} \mathbf{1}_{\sigma^{c}}\right) .
$$

Now, using the above results, we obtain from Eq. (2.15)

$$
\begin{aligned}
& \int_{\sigma_{\varepsilon_{n, k}}}\left|\psi_{k+n}^{(n)}(\lambda)\right|^{2}\left[-\sin ^{2} \frac{\theta}{2}+\cot \frac{z-\lambda}{2}(\sin \lambda-\sin z)\right] d \lambda \\
& \quad+\int_{\sigma_{\varepsilon_{n, k}}}\left|\psi_{k+n}^{(n)}(\lambda)\right|^{2} \sin z \frac{\sqrt{\sin ^{2} z / 2-\sin ^{2} \theta / 2}}{\sin z / 2} \cot \frac{\lambda-z}{2} d \lambda=O\left(\varepsilon_{n, k}|\operatorname{dist}(z, \sigma)|^{-3}\right) .
\end{aligned}
$$

Denote $R^{(n)}$ the analog of the resolvent for the matrix $C^{(n)}$ :

$$
R^{(n)}=i \frac{C^{(n)}+e^{i z}}{C^{(n)}-e^{i z}}
$$

Then by the spectral theorem

$$
\begin{gathered}
R_{n+k, n+k}^{(n)}(z)=\int\left|\psi_{k+n}^{(n)}(\lambda)\right|^{2} \cot \frac{\lambda-z}{2} d \lambda, \\
C_{n+k, n+k}^{(n)}+\cos z=\int\left|\psi_{k+n}^{(n)}(\lambda)\right|^{2} \cot \frac{\lambda-z}{2}(\sin \lambda-\sin z) d \lambda .
\end{gathered}
$$

The above relations combined with Eq. (2.14) allow us to derive from Eq. (2.20) that

$$
R_{n+k, n+k}^{(n)}(z)=\frac{C_{n+k, n+k}^{(n)}+\cos z+\sin ^{2} \theta / 2+O\left(\varepsilon_{n, k}|\operatorname{dist}(z, \sigma)|^{-3}\right)}{2 \cos (z / 2) \sqrt{\sin ^{2} z / 2-\sin ^{2} \theta / 2}} .
$$

Integrating the product $e^{i z} R_{n+k, n+k}^{(n)}(z)$ over the contour $\mathfrak{L}_{\delta}=\{z \mid \operatorname{dist}(z, \sigma)=\delta\}$ for some $\delta$ we obtain from Eq. (2.22)

$$
\oint_{\mathfrak{L}_{\delta}} e^{i z} R_{n+k, n+k}^{(n)}(z) d z=\oint_{\mathfrak{L}_{\delta}} e^{i z} \frac{C_{n+k, n+k}^{(n)}+\cos z+\sin ^{2} \theta / 2}{2 \cos (z / 2) \sqrt{\sin ^{2} z / 2-\sin ^{2} \theta / 2}} d z+O\left(\varepsilon_{n, k} \delta^{-7 / 2}\right) .
$$


The Cauchy theorem and the spectral theorem yield

$$
\oint_{\mathfrak{L}_{\delta}} e^{i z} R_{n+k, n+k} d z=-4 i \pi C_{n+k, n+k}^{(n)} .
$$

Since the integrand in the rhs of Eq. (2.23) is analytic outside $\sigma$ the integral does not depend on $\delta$, therefore we can compute this integral with $\delta \rightarrow 0$.

$$
\begin{aligned}
I\left(C_{n+k, n+k}^{(n)}\right) & :=\lim _{\delta \rightarrow 0} \oint_{\mathfrak{L}_{\delta}} e^{i z} \frac{C_{n+k, n+k}^{(n)}+\cos z+\sin ^{2} \theta / 2}{2 \cos (z / 2) \sqrt{\sin ^{2} z / 2-\sin ^{2} \theta / 2}} d z \\
& =-i \int_{-\theta}^{\theta} \cos \mu \frac{C_{n+k, n+k}^{(n)}+\cos \mu+\sin ^{2} \theta / 2}{\cos (\mu / 2) \sqrt{\sin ^{2} \theta / 2-\sin ^{2} \mu / 2}} d \mu .
\end{aligned}
$$

Then, using the change of the variable $\sin \mu / 2=t$ with Eq. (2.19) we get

$$
I\left(C_{n+k, n+k}^{(n)}\right)=-4 i \pi C_{n+k, n+k}^{(n)}-\frac{2 i \pi}{\cos \theta / 2}\left(C_{n+k, n+k}^{(n)}-\cos ^{2} \theta / 2\right) .
$$

Combining Eqs. (2.17), (2.24), and (2.25) we have

$$
C_{n+k, n+k}^{(n)}=\cos ^{2} \frac{\theta}{2}+O\left(\varepsilon_{n, k}\right)
$$

Then in view of Eq. (2.22) we obtain for the "resolvent"

$$
R_{n+k, n+k}^{(n)}(z)=\frac{1+\cos z+O\left(\varepsilon_{n, k}|\operatorname{dist}(z, \sigma)|^{-3}\right)}{2 \cos z / 2 \sqrt{\sin ^{2} z / 2-\sin ^{2} \theta / 2}} .
$$

Similarly, integrating the product $e^{2 i z} R_{n+k, n+k}^{(n)}(z)$ over the contour $\mathfrak{L}_{\delta}$ and taking $\delta \rightarrow 0$, we get

$$
\begin{array}{r}
-4 i \pi\left(C^{(n)}\right)_{n+k, n+k}^{2}=-i \int_{-\theta}^{\theta} \frac{2 \cos \mu / 2 \cos 2 \mu}{\sqrt{\sin ^{2} \theta / 2-\sin ^{2} \mu / 2}} d \mu+O\left(\varepsilon_{n, k}\right) \\
=-4 i \pi\left(3 \sin ^{4} \frac{\theta}{2}-4 \sin ^{2} \frac{\theta}{2}+1+O\left(\varepsilon_{n, k}\right)\right) .
\end{array}
$$

From Eq. (1.4) we have

$$
\begin{aligned}
\left(C^{(n)}\right)_{m, m}^{2}= & C_{m, m-1}^{(n)} C_{m-1, m}^{(n)}+C_{m, m}^{(n)} C_{m, m}^{(n)}+C_{m, m+1}^{(n)} C_{m+1, m}^{(n)} \\
& =C_{m, m}^{(n)} C_{m-1, m-1}^{(n)}+C_{m, m}^{(n)} C_{m, m}^{(n)}+C_{m, m}^{(n)} C_{m+1, m+1}^{(n)}-\alpha_{m-1}^{(n)} \alpha_{m+1}^{(n)}-\alpha_{m}^{(n)} \alpha_{m+2}^{(n)} .
\end{aligned}
$$

Taking into account Eqs. (2.26) and (2.28), we obtain from Eq. (2.29)

$$
\left\{\begin{array}{c}
\alpha_{n+k-1}^{(n)} \alpha_{n+k+1}^{(n)}+\alpha_{n+k}^{(n)} \alpha_{n+k+2}^{(n)}=2 \cos ^{2} \frac{\theta}{2}+O\left(\varepsilon_{n, k}\right) \\
\alpha_{n+k-1}^{(n)} \alpha_{n+k}^{(n)}=-\cos ^{2} \frac{\theta}{2}+O\left(\varepsilon_{n, k}\right)
\end{array} .\right.
$$

To solve the system we multiply the first equation in Eq. (2.30) by $\alpha_{n+k}^{(n)} \alpha_{n+k+1}^{(n)}$. By the definition of the Verblunsky coefficients $\left|\alpha_{j}^{(n)}\right| \leq 1$, hence using the second equation of Eq. (2.30) we obtain

$$
\left(\alpha_{n+k+1}^{(n)}\right)^{2}+\left(\alpha_{n+k}^{(n)}\right)^{2}=2 \cos ^{2} \frac{\theta}{2}+O\left(\varepsilon_{n, k}\right)
$$

Finally, combining the above relations, we obtain Eq. (2.1). 
Remark 11: If the support of the equilibrium measure is the whole circle we can use function $\phi(\mu)=\cot \frac{z-\mu}{2} \rho^{-1}(\mu)$ in Eq. (2.16) and obtain similarly to Eq. (2.26)

$$
C_{n+k, n+k}^{(n)}=O\left(n^{-1 / 2} \log n+\frac{|k|}{n}\right)
$$

Proof of Theorem 4. Let

$$
\alpha_{n+m}^{(n)}=(-1)^{n+m} s \cos \left(\frac{\theta}{2}+x_{n+m}^{(n)}\right), \quad \rho_{n+m}^{(n)}=\sin \left(\frac{\theta}{2}+x_{n+m}^{(n)}\right),
$$

where $s= \pm 1$ and $x_{n+m} \in[-\pi, \pi)$. We have already a priori approximate Eq. (2.1)

$$
x_{n+m}^{(n)}=\underline{O}\left(n^{-1 / 4} \log ^{1 / 2} n+\left|\frac{m}{n}\right|^{1 / 2}\right) .
$$

In this part of the paper we use the string equation for unitary matrix models (1.13). We consider these equations as a system of nonlinear equations for $x_{n+m}^{(n)}$. Denote

$$
M^{(n)}=\frac{C^{(n)}+C^{(n) *}}{2}, \quad L^{(n)}=\frac{C^{(n)}-C^{(n) *}}{2} .
$$

Equation (1.13) can be rewritten as

$$
\left\{L^{(n)} V^{\prime}\left(M^{(n)}\right)\right\}_{n+k, n+k-1}=(-1)^{n+k} \frac{n+k}{n} \frac{\alpha_{n+k}^{(n)}}{\rho_{n+k}^{(n)}} .
$$

To simplify notations we set

$$
\begin{gathered}
\tilde{M}_{j, k}=M_{n+j, n+k}^{(n)}, \quad \widetilde{L}_{j, k}=L_{n+j, n+k}^{(n)}, \quad \tilde{\alpha}_{k}=\alpha_{n+k}^{(n)}, \quad \tilde{x}_{k}=x_{n+k}^{(n)}, \\
a=\cos ^{2} \frac{\theta}{2}, \quad b=\cos \frac{\theta}{2} \sin \frac{\theta}{2}, \quad c=\sin ^{2} \frac{\theta}{2} .
\end{gathered}
$$

and define $M^{\star}$ as a double-infinite matrix with entries

$$
M_{j, k}^{\star}=\delta_{j, k} a+\frac{1}{2}\left(\delta_{j-2, k}+\delta_{j, k-2}\right) c .
$$

The spectrum and the generalized eigenvectors of the matrix can be parameterized as

$$
M^{\star} \sim\left\{a+c \cos 2 \lambda, \lambda \in[-\pi, \pi] ; v_{k}=\frac{1}{\sqrt{2 \pi}} e^{i k \lambda}\right\} .
$$

From now we consider matrix $\tilde{M}$ as a double-infinite one and redefine it for $j, k<-n$ equal to $M^{\star}$. It is convenient for us to extend the function $V^{\prime}$ from $[-1,1]$ to $\mathbb{R}$ in such a way to obtain the function from $\mathcal{L}_{2}(\mathbb{R})$ with the third derivative bounded in $\mathcal{L}_{2}(\mathbb{R})$. Denoting $\widehat{V}^{\prime}$ the Fourier transform of the extension we have for $x \in[0,2 \pi]$

$$
V^{\prime}(x)=\frac{1}{2 \pi} \int_{-\infty}^{\infty} \widehat{V}^{\prime}(\xi) e^{i x \xi} d \xi .
$$

Using Eq. (2.40), $V^{\prime}\left(M^{(n)}\right)$ can be represented

$$
V^{\prime}(\tilde{M})_{i, j}=\frac{1}{2 \pi} \int_{-\infty}^{\infty} \widehat{V}^{\prime}(\xi)\left\{e^{i \xi \tilde{M}}\right\}_{i, j} d \xi
$$


Applying the Duhamel formula twice to matrices $\widetilde{M}$ and $M^{*}$, we obtain

$$
\begin{aligned}
e^{i \xi \widetilde{M}}= & e^{i \xi M^{\star}}+i \xi \int_{0}^{1} e^{i \xi M^{\star} t}(\delta M) e^{i \xi M^{\star}(1-t)} d t \\
& -\xi^{2} \int_{0}^{1} d t \int_{0}^{1-t} d s e^{i \xi M^{\star} t}(\delta M) e^{i \xi \widetilde{M} s}(\delta M) e^{i \xi M^{\star}(1-t-s)}, \quad \text { for } \delta M=\widetilde{M}-M^{\star}
\end{aligned}
$$

Denote for $d=0, \pm 1, \pm 2$

$$
\begin{array}{ll}
\hat{f}(\xi)=\left\{e^{i \xi \widetilde{M}}\right\}_{k+d, k-1}, & \hat{f}^{0}(\xi)=\left\{e^{i \xi M^{*}}\right\}_{k+d, k-1}, \\
\hat{f}^{1}(\xi)=\left\{i \xi \int_{0}^{1} e^{i \xi M^{* t}}(\delta M) e^{i \xi M^{*}(1-t)} d t\right\}_{k+d, k-1}, \widehat{r}(\xi ; d)=\hat{f}(\xi)-\hat{f}^{0}(\xi)-\hat{f}^{1}(\xi) .
\end{array}
$$

Now we find the first two terms in Eq. (2.42).

$$
\begin{aligned}
\hat{f}^{0}(\xi)=\left\{e^{i \xi M^{*}}\right\}_{k+d, k-1}=\frac{1}{2 \pi} \int_{-\pi}^{\pi} e^{i \xi(a+c \cos 2 \phi)} e^{i(k+d) \phi} e^{-i(k-1) \phi} d \phi & \\
= & \frac{1}{2 \pi} \int_{-\pi}^{\pi} e^{i \xi(a+c \cos 2 \phi)} \cos (d+1) \phi d \phi .
\end{aligned}
$$

Let $\left\{v_{k}\right\}$ be the coefficients of the Fourier series for the function $V^{\prime}(a+c \cos 2 \phi)$, i.e.,

$$
v_{k}=\frac{1}{\pi\left(1+\delta_{k, 0}\right)} \int_{-\pi}^{\pi} V^{\prime}(a+c \cos 2 \phi) \cos k \phi d \phi .
$$

Note that $v_{2 l+1}=0$, for any integer $l$. By the spectral theorem we obtain

$$
\begin{aligned}
\left\{\widetilde{L} V^{\prime}\left(M^{\star}\right)\right\}_{k, k-1}=\sum_{d=0, \pm 1, \pm 2} \widetilde{L}_{k, k+d} \frac{1}{2 \pi} \int_{-\pi}^{\pi} V^{\prime}(a+c & \cos 2 \phi) \cos (d+1) \phi d \phi \\
& =\frac{\widetilde{L}_{k, k+1}}{c}+\left(\widetilde{L}_{k, k+1}+\widetilde{L}_{k, k-1}\right) v_{0}
\end{aligned}
$$

where we have used the relation

Proposition 12: Under conditions C1-C3 for $v_{k}$ defined in Eq. (2.45)

$$
v_{2}=2 v_{0}+\frac{2}{c}
$$

For the second term in Eq. (2.42) we have

$$
\widehat{f}^{1}(\xi)=i \xi \sum_{l=-\infty}^{\infty} \sum_{d_{1}=-2}^{2} \int_{0}^{1}\left\{e^{i t \xi M^{*}}\right\}_{k+d, k+l} \delta M_{k+l, k+l+d_{1}}\left\{e^{i(1-t) \xi M^{*}}\right\}_{k+l+d_{1}, k-1} d t
$$

and by the spectral theorem and Eq. (2.39) we have

$$
\widehat{f}^{1}(\xi)=\sum_{l=-\infty}^{\infty} \sum_{d_{1}=-2}^{2} \delta M_{k+l, k+l+d_{1}} \frac{e^{i \xi a}}{4 c \pi^{2}} \int_{-\pi}^{\pi} \int_{-\pi}^{\pi} \frac{e^{i \xi c \cos 2 \phi}-e^{i \xi c \cos 2 \psi}}{\cos 2 \phi-\cos 2 \psi} e^{i\left((l-d) \phi+\left(l+d_{1}+1\right) \psi\right)} d \phi d \psi .
$$

Hence, integrating Eq. (2.47) with $\widehat{V}^{\prime}$ we obtain the second term in Eq. (2.35) in the form

$$
\frac{1}{2 \pi} \int_{-\infty}^{\infty} \widehat{V}^{\prime}(\xi) \widehat{f}^{1}(\xi) d \xi=\sum_{l=-\infty}^{\infty} \sum_{d_{1}=-2}^{2} \delta M_{k+l, k+l+d_{1}} \mathbf{B}_{l-d, l+d_{1}+1}
$$

where

$$
\mathbf{B}_{\alpha, \beta}=\frac{1}{4 \pi^{2} c} \int_{-\pi}^{\pi} d u \int_{-\pi}^{\pi} d v \frac{V^{\prime}(a+c \cos 2 u)-V^{\prime}(a+c \cos 2 v)}{\cos 2 u-\cos 2 v} e^{i(\alpha u+\beta v)} .
$$


Below we need some properties of these coefficients.

Proposition 13: For any function $V$ satisfying condition $C 3$ coefficient $\mathbf{B}_{\alpha, \beta}$ depends only on $|\alpha|$ $+|\beta|$, i.e. $\mathbf{B}_{\alpha, \beta}=\mathbf{B}_{|\alpha|+|\beta|}$, equals to zero when $\alpha$ or $\beta$ is odd and for any positive $\gamma$

$$
\mathbf{B}_{2 \gamma}=\frac{1}{c} \sum_{j-\gamma \in 2 \mathbb{N}+1} v_{2 j}=\underline{O}\left(\gamma^{-5 / 2}\right) .
$$

Since $\delta M_{k, l}$ are bounded, the proposition yields that the series in the rhs of Eq. (2.48) is convergent. Using Eqs. (2.35), (2.46), and (2.48), we obtain the equation valid for any $k$

$$
\begin{array}{r}
\frac{\widetilde{L}_{k, k+1}}{c}+\left(\widetilde{L}_{k, k+1}+\widetilde{L}_{k, k-1}\right) v_{0}+\sum_{d, d_{1}=-2}^{2} \sum_{l=-\infty}^{\infty} \widetilde{L}_{k, k+d} \mathbf{B}_{l-d, l+d_{1}+l} \delta M_{k+l, k+l+d_{1}}+r_{k}^{(n)} \\
=(-1)^{k} \frac{\widetilde{\alpha}_{k}}{\widetilde{\rho}_{k}}\left(1+\frac{k}{n}\right)
\end{array}
$$

for

$$
r_{k}^{(n)}=\frac{1}{2 \pi} \int_{-\infty}^{\infty} \widehat{V}^{\prime}(\xi) \sum_{d=-2}^{2} \widehat{r}(\xi ; d) d \xi
$$

Set

$$
S_{k}=\sum_{j=-10}^{10} \widetilde{x}_{k+j}^{2} .
$$

Then $\widetilde{L}_{k, j}$ and $\delta M_{k, j}$ can be written up to the errors $\underline{O}\left(S_{k}\right)$ as

$$
\begin{array}{ll}
\delta M_{k, k-2}=-\frac{b}{2}\left(\widetilde{x}_{k-1}+\tilde{x}_{k}\right), & \widetilde{L}_{k, k-2}=(-1)^{n+k}\left(\frac{c}{2}+\frac{b}{2}\left(\widetilde{x}_{k-1}+\tilde{x}_{k}\right)\right) \\
\delta M_{k, k-1}=(-1)^{n+k-1} s \frac{c}{2}\left(\widetilde{x}_{k-1}-\widetilde{x}_{k+1}\right), & \widetilde{L}_{k, k-1}=-s b-s a \widetilde{x}_{k}+s \frac{c}{2}\left(\widetilde{x}_{k-1}+\widetilde{x}_{k+1}\right) \\
\delta M_{k, k}=b\left(\widetilde{x}_{k}+\widetilde{x}_{k+1}\right), & \widetilde{L}_{k, k}=0 \\
\delta M_{k, k+1}=(-1)^{n+k} s \frac{c}{2}\left(\widetilde{x}_{k}-\widetilde{x}_{k+2}\right), & \widetilde{L}_{k, k+1}=s b+s a \widetilde{x}_{k+1}-s \frac{c}{2}\left(\widetilde{x}_{k}+\widetilde{x}_{k+2}\right) \\
\delta M_{k, k+2}=-\frac{b}{2}\left(\widetilde{x}_{k+1}+\tilde{x}_{k+2}\right), & \widetilde{L}_{k, k+2}=(-1)^{n+k+1}\left(\frac{c}{2}+\frac{b}{2}\left(\widetilde{x}_{k+1}+\widetilde{x}_{k+2}\right)\right)
\end{array}
$$

Replacing $\widetilde{L}_{i, j}$ and $\delta M_{i, j}$ by their first order approximations and reminders we get a system of equations with respect to $\tilde{x}_{j}$.

Lemma 14: Under conditions C1-C3 equations (2.51) for $\left\{\widetilde{x}_{k}\right\}_{|k| \leq \varepsilon_{1} n}$ have the form

$$
\sum_{l} A_{k-l} \widetilde{x}_{l}=\frac{b}{c} \frac{k}{n}+R_{k}
$$

and the Toplitz matrix A has the symbol

$$
\delta(\phi)=\sum A_{l} e^{i l \phi}=\frac{\sqrt{1-c \cos ^{2}(\phi / 2)}}{\pi \sqrt{2}} P\left(-2 \arcsin \left(\sin \frac{\theta}{2} \cos \frac{\phi}{2}\right)\right) .
$$

Reminder $R_{k}$ satisfies the bound

$$
R_{k} \leq C\left(\frac{k^{2}}{n^{2}}+S_{k}+\sum S_{k+2 l}|l|^{-5 / 2}\right)+r_{k}^{(n)}
$$


where

$$
\begin{gathered}
\left|r_{k}^{(n)}\right|^{2} \leq C \int_{0}^{\infty} e^{-y} y^{5}\left\|r_{k, y}\right\|_{2}^{2} d y, \\
r_{k, y}(x)=\min \left\{y^{-1} \sum_{s=-\infty}^{\infty}\left|R_{2 s, 0}^{\star}\right|^{2} S_{k+2 s}, \quad \operatorname{dist}^{-1}(z,[-1,1])+y^{-1} \mathfrak{J} R_{0,0}^{\star}\right\},
\end{gathered}
$$

where $\|\cdot\|_{2}$ means the $L_{2}(\mathbb{R})$ norm, and $R^{\star}(z)=\left(M^{\star}-z I\right)^{-1}, \quad z=-x+i y$.

Equations (2.53) are true only for $|k| \leq \varepsilon_{1} n$. But we extend them to all $k \in \mathbb{Z}$ redefining Eq. (2.53) by the identities. All bounds for the reminders will be valid because the reminder for $k \sim$ $n$ is of order 1 .

Now we start from the bound (2.1) and using the perturbation theory method obtain Eq. (1.12) in a few steps. Suppose we have proved the bound

$$
\widetilde{x}_{m}=\underline{O}\left(\log ^{\beta} n\left(n^{-\alpha}+|m / n|^{\gamma}\right)\right) .
$$

Note that on the first step we have it with $\alpha=1 / 4, \beta=1 / 2, \gamma=1 / 2$. This bound yields that

$$
S_{k+2 s} \leq \log ^{2 \beta} n\left(n^{-2 \alpha}+n^{-2 \gamma}+\min \left\{|k / n|^{2 \gamma}+|s / n|^{2 \gamma}, 1\right\}\right) .
$$

To estimate the reminder $R_{k}$ we must estimate $r_{k, y}(x)$ first. For this aim we need some properties of $R^{\star}$. From the spectral theorem we have

$$
R_{j, l}^{\star}=G_{j-l}:=\frac{1}{2 \pi} \int_{0}^{2 \pi} \frac{e^{i(j-l) \phi} d \phi}{a-z+c \cos 2 \phi} .
$$

Using this representation we will estimate the sums in the rhs of Eq. (2.56).

Proposition 15: Let $G_{l}$ be defined in Eq. (2.59). Then for any integer $p$

$$
\int_{-\infty}^{\infty}\left(\sum_{s=0}^{\infty}\left|G_{2 s}(-x+i y)\right|^{2} s^{p}\right)^{2} d x \leq C_{p} \frac{\max \{1, \log 1 / y\}}{y^{2 p+2}} .
$$

To estimate $r_{k}^{(n)}$ we use the second bound from the rhs of Eq. (2.56) for small $y$ and the first bound for sufficiently big $y$. Combining Eq. (2.56) with Eq. (2.58) we get

$$
\begin{aligned}
r_{k, y}(x) \leq & \frac{C_{\alpha, \beta, \gamma} \log ^{2 \beta} n}{y} . \\
& \begin{cases}\sum_{s=0}^{\infty}\left|G_{2 s}(-x+i y)\right|^{2}\left(n^{-2 \alpha}+n^{-2 \gamma}+\min \left\{|k / n|^{2 \gamma}, 1\right\}+|s / n|^{2 \gamma}\right), & y \geq n^{-\delta} \\
\mathfrak{J} G_{0}, & y \leq n^{-\delta},\end{cases}
\end{aligned}
$$

where $\delta$ we will choose on every step. Integrating the square of the last equation over $x$ and using Eqs. (2.60) and (3.25), we obtain

$$
\left\|r_{k, y}(x)\right\|_{2}^{2} \leq \frac{C_{\alpha, \beta, \gamma}^{\prime} \log ^{4 \beta+1} n}{y^{4}} \cdot \begin{cases}\left(n^{-4 \alpha}+n^{-4 \gamma}+\min \left\{|k / n|^{4 \gamma}, 1\right\}+(n y)^{-4 \gamma}\right), & y \geq n^{-\delta} \\ y^{2} \log 1 / y, & y \leq n^{-\delta}\end{cases}
$$

The last bound and Eq. (2.56) imply

$$
\left|r_{k}^{(n)}\right|^{2} \leq C_{\alpha, \beta, \gamma, \delta} \log ^{4 \beta+2} n\left(n^{-4 \delta}+n^{-4 \alpha}+n^{-4 \gamma}+\min \left\{|k / n|^{4 \gamma}, 1\right\}+n^{-4 \gamma+\delta(4 \gamma-2)}\right) .
$$


Now we estimate $R_{k}$. The term $\sum_{l} S_{k+2 l} l^{-5 / 2}$ can be estimated using Eq. (2.58).

$$
\begin{aligned}
\sum_{l} S_{k+2 l} l^{-5 / 2} & =\sum_{l \leq n}+\sum_{l \geq n} \\
& \leq C_{\alpha, \beta, \gamma, \delta}^{\prime} \log ^{2 \beta} n\left(n^{-2 \alpha}+n^{-2 \gamma}+\min \left\{|k / n|^{2 \gamma}, 1\right\}+n^{-3 / 2}\right) .
\end{aligned}
$$

Combining the above relations finally we can estimate the reminder $R_{k}$

$$
R_{k}=\underline{O}\left(k^{2} / n^{2}+n^{-3 / 2}+\log ^{2 \beta+1} n\left(n^{-2 \delta}+n^{-2 \alpha}+\min \left\{|k / n|^{2 \gamma}, 1\right\}+n^{-2 \gamma+\delta(2 \gamma-1)}\right)\right) .
$$

To find $\tilde{x}_{j}$ we find the inverse matrix of $\left\{A_{k-l}\right\}_{k, l=-\infty}^{\infty}$. By the spectral theorem

$$
\left(A^{-1}\right)_{k, l}=\left(A^{-1}\right)_{k-l}=\frac{1}{2 \pi} \int_{-\pi}^{\pi} \frac{e^{i(k-l) \phi}}{\delta(\phi)} d \phi .
$$

From the conditions $\mathrm{C} 1-\mathrm{C} 2 P(\lambda)>C>0$ on the interval $[-\theta, \theta]$, therefore the solution of Eq. (2.53) can be found as

$$
\tilde{x}_{k}=\frac{b}{c} \sum_{m}\left(\frac{m+k}{n}+R_{m+k}\right)\left(A^{-1}\right)_{m}=\frac{\pi \sqrt{2}}{P(\theta) \sin \theta / 2} \frac{k}{n}+\sum_{m} R_{m+k}\left(A^{-1}\right)_{m}
$$

Since $P(\lambda)$ is a twice differentiable function we have $\sum_{m}\left|A_{m}^{-1}\right|^{2} m^{4} \leq C$. Therefore

$$
\begin{aligned}
\left|\sum_{m} R_{m+k} A_{m}^{-1}\right| & / \log ^{2 \beta+1} n \\
=\underline{O}\left(\sum_{m}\left|A_{m}^{-1}\right|\right. & \left.\left(n^{-2 \delta}+n^{-2 \alpha}+n^{-3 / 2}+n^{-2 \gamma+\delta(2 \gamma-1)}+\min \left\{\frac{k^{2}}{n^{2}}, 1\right\}+\min \left\{\left|\frac{k}{n}\right|^{2 \gamma}, 1\right\}\right)\right) \\
& +\underline{O}\left(\sum_{|m| \leq n}\left|A_{m}^{-1}\right|\left(\left|\frac{m}{n}\right|^{2}+\left|\frac{m}{n}\right|^{2 \gamma}\right)\right)+\underline{O}\left(\sum_{|m| \geq n}\left|A_{m}^{-1}\right|\right) \\
= & \underline{O}\left(n^{-2 \delta}+n^{-2 \alpha}+n^{-3 / 2}+n^{-2 \gamma+\delta(2 \gamma-1)}+\min \left\{\frac{k^{2}}{n^{2}}, 1\right\}+\min \left\{\left|\frac{k}{n}\right|^{2 \gamma}, 1\right\}\right),
\end{aligned}
$$

where we use

$$
\sum_{|m| \leq n}\left|A_{m}^{-1}\right|\left|\frac{m}{n}\right|^{2 \gamma}=\sum_{|m| \leq n}\left|A_{m}^{-1}\right| m^{2} \cdot\left|\frac{m}{n}\right|^{2 \gamma} m^{-2} \leq C n^{-3 / 2} .
$$

Using the last inequality for $\tilde{x}$ with $\alpha=1 / 4, \beta=1 / 2, \gamma=1 / 2, \delta=1 / 4$ we obtain bound (2.57) with $\alpha=1 / 2, \beta=2, \gamma=1$. Taking $\delta=1 / 2$ we have $\alpha=1, \beta=5, \gamma=2$ and finally with $\delta=2 / 3$ we get $\alpha=4 / 3, \beta=11, \gamma=2$.

$$
\tilde{x}_{k}=\frac{\pi \sqrt{2}}{P(\theta) \sin \theta / 2} \frac{k}{n}+\underline{O}\left(\log ^{11} n\left(n^{-4 / 3}+\frac{k^{2}}{n^{2}}\right)\right) .
$$

\section{AUXILIARY RESULTS.}

Proof of Proposition 2. To prove Eq. (1.9) we denote

$$
D_{\theta}=\{z:|z|=1, \arg z \in[-\theta, \theta]\} .
$$

Consider two functions defined on $D_{\theta}$

$$
F\left(e^{i \lambda}\right)=\frac{i-(V(\cos \lambda))^{\prime}}{2 \pi i}, \quad G\left(e^{i \lambda}\right)=\rho(\lambda) .
$$


From Eq. (1.6) we obtain

$$
F(\xi)=\frac{1}{\pi i} v \cdot p \cdot \int_{\mathfrak{L}} \frac{G(\zeta)}{\zeta-\xi} d \zeta
$$

where $\mathfrak{L}$ is $D_{\theta}$ with the direction from $\zeta_{0}=e^{-i \theta}$ to $\overline{\zeta_{0}}$. Then by the standard method (see Ref. 12) we get

$$
G(z)=\frac{X(z)}{2 \pi i} v \cdot p \cdot \int_{\mathfrak{L}} \frac{F(\zeta)}{\zeta-z} \frac{d \zeta}{X^{+}(\zeta)}
$$

where

$$
X(z)=\sqrt{\left(z-\zeta_{0}\right)\left(z-\overline{\zeta_{0}}\right)}
$$

It is easy to see that $X^{+}\left(e^{i \lambda}\right)=\sqrt{2} e^{i \lambda / 2} \chi(\lambda)$. Using the identity (2.19) we obtain that $v \cdot p \cdot \int_{\mathfrak{L}} \frac{d \zeta}{\left(\zeta-\zeta_{1}\right) X^{+}(\zeta)}=0$. From the last relation and Eq. (3.1) we obtain Eq. (1.9).

Proof of Lemma 8. Consider the function

$$
F(\lambda)=\frac{1}{4 \pi^{2}} \int_{-\theta}^{\theta} \cot \frac{\lambda-\mu}{2} \frac{P(\mu)(\cos \mu-\cos \theta)-P(\lambda)(\cos \lambda-\cos \theta) \frac{\cos \mu / 2}{\cos \lambda / 2}}{\sqrt{\cos \mu-\cos \theta}} d \mu .
$$

We prove first that $F$ coincides with $V^{\prime}$ on $\sigma$. For this aim we compute the integral

$$
\begin{aligned}
I(\lambda)=v \cdot p \cdot \int_{-\theta}^{\theta} \cot \frac{\lambda-\mu}{2} \frac{\cos (\mu / 2) d \mu}{\chi(\mu)}=v \cdot p \cdot \int_{-\theta}^{\theta} \frac{\sin \lambda}{\cos \mu-\cos \lambda} \frac{\cos (\mu / 2) d \mu}{\chi(\mu)} \\
=\frac{\sin \lambda}{\sqrt{2}} v \cdot p \cdot \int_{-\sin (\theta / 2)}^{\sin (\theta / 2)} \frac{d t}{\left(\sin ^{2} \lambda / 2-t^{2}\right) \sqrt{\sin ^{2} \theta / 2-t^{2}}}=2 \pi \frac{\cos (\lambda / 2) \operatorname{sign} \lambda}{\sqrt{\cos \theta-\cos \lambda}} \mathbf{1}_{[-\theta, \theta]^{c}},
\end{aligned}
$$

where we have used Eq. (2.19). This relation and Eq. (1.6) imply that $F(\lambda)=(V(\cos \lambda))^{\prime}$ for $\lambda \in \sigma$. Since $P$ is a twice differentiable function, we obtain that $F$ is a differentiable function. Then

$$
F(\lambda)-\left.V^{\prime}(\lambda)\right|_{\lambda=\theta-0}=0 \Rightarrow F^{\prime}(\lambda)-\left.V^{\prime \prime}(\lambda)\right|_{\lambda=\theta-0}=0 \Rightarrow F^{\prime}(\theta)=V^{\prime \prime}(\theta)
$$

The function $F-V^{\prime}$ is a differentiable on $\sigma_{\varepsilon}$ and equals 0 with its first derivative on $\sigma$. Hence we have $F(\lambda)-(V(\cos \lambda))^{\prime}=O(|\cos \lambda-\cos \theta|)$ for $\lambda \in \sigma_{\varepsilon} \backslash \sigma$.

Proof of Proposition 6. From the identity

$$
\int_{-\pi}^{\pi}\left(\chi_{k}^{(n)}(\lambda) \overline{\chi_{k-1}^{(n)}(\lambda)} e^{-n V(\cos \lambda)}\right)^{\prime} d \lambda=0,
$$

and orthogonality $\left\langle\frac{d}{d \lambda} \chi_{k-1}^{(n)}(\lambda), \chi_{k}^{(n)}(\lambda)\right\rangle=0$ we get

$$
\int_{0}^{2 \pi} \sin \lambda V^{\prime}(\cos \lambda) \chi_{k}^{(n)}(\lambda) \overline{\chi_{k-1}^{(n)}(\lambda)} e^{-n V(\cos \lambda)} d \lambda=-\frac{1}{n}\left\langle\frac{d}{d \lambda} \chi_{k}^{(n)}, \chi_{k-1}^{(n)}\right\rangle .
$$

To compute the rhs of Eq. (3.5) we use the definition of orthogonal polynomials (1.1) and some properties of polynomials $P_{k}^{(n)}$ and $Q_{k}^{(n)}$. We assume that $k$ is odd. For even $k$ the proof is similar. 
From the definition of $\chi_{k}^{(n)}$ we obtain

$$
\begin{aligned}
I_{k, n}:=\left\langle\frac{d \chi_{2 k+1}^{(n)}(\lambda)}{d \lambda}, \chi_{2 k}^{(n)}(\lambda)\right\rangle & =\left\langle\frac{d}{d \lambda}\left(e^{-i k \lambda} P_{2 k+1}^{(n)}(\lambda)\right), e^{-i k \lambda} Q_{2 k}^{(n)}(\lambda)\right\rangle \\
= & -i k\left\langle e^{-i k \lambda} P_{2 k+1}^{(n)}(\lambda), e^{-i k \lambda} Q_{2 k}^{(n)}(\lambda)\right\rangle+\left\langle\frac{d}{d \lambda} P_{2 k+1}^{(n)}(\lambda), Q_{2 k}^{(n)}(\lambda)\right\rangle .
\end{aligned}
$$

From the definition of $P_{k}^{(n)}$ we have

$$
P_{k}^{(n)}(\lambda) \perp e^{i m \lambda}, m=0, \ldots, k-1 ; \quad Q_{k}^{(n)}(\lambda) \perp e^{i m \lambda}, m=1, \ldots, k .
$$

Therefore the first term in the above relation is zero. Hence

$$
I_{k, n}=i(2 k+1) c_{2 k+1,2 k+1}^{(n)}\left\langle e^{i(2 k+1) \lambda}, Q_{2 k}^{(n)}(\lambda)\right\rangle .
$$

Now using Eq. (3.6) one more time and equality $\left\|P_{k}^{(n)}\right\|=1$ we obtain

$$
\begin{aligned}
I_{k, n}=i(2 k+1)\left\langle P_{2 k+1}^{(n)}(\lambda)-c_{2 k+1,0}^{(n)}, Q_{2 k}^{(n)}(\lambda)\right\rangle & \\
=-i(2 k+1) c_{2 k+1,0}^{(n)}\left\langle 1, Q_{2 k}^{(n)}(\lambda)\right\rangle & =-i(2 k+1) c_{2 k+1,0}^{(n)}\left\langle P_{2 k}^{(n)}(\lambda), e^{i 2 k \lambda}\right\rangle \\
& =-i(2 k+1) \frac{c_{2 k+1,0}^{(n)}}{c_{2 k, 2 k}^{(n)}}=-i(2 k+1) \frac{\alpha_{2 k+1}^{(n)}}{\rho_{2 k+1}^{(n)}}
\end{aligned}
$$

Relations (3.5) and (3.7) give us Eq. (1.13).

Proof of Proposition 12: We prove the proposition, using Eq. (1.6). Consider the difference $v_{2}-2 v_{0}$ and its integral representation.

$$
v_{2}-2 v_{0}=\frac{1}{\pi} \int_{-\pi}^{\pi} V^{\prime}(a+c \cos 2 \phi)(\cos 2 \phi-1) d \phi .
$$

Changing the variables with $\sin \frac{\lambda}{2}=\sin \frac{\theta}{2} \sin \phi$, we get

$$
v_{2}-2 v_{0}=\frac{1}{\pi c^{3 / 2}} \int_{\sigma}-V^{\prime}(\cos \lambda) \sin \lambda \frac{\sin \lambda / 2}{\cos \phi} d \lambda
$$

And finally, using Eq. (1.6), we obtain

$$
v_{2}-2 v_{0}=\frac{1}{c \pi} \int_{\sigma} d \mu \rho(\mu) v \cdot p \cdot \int_{\sigma} \cot \frac{\lambda-\mu}{2} \frac{\sin \lambda / 2 d \lambda}{\sqrt{\sin ^{2} \theta / 2-\sin ^{2} \lambda / 2}}=\frac{2}{c} \int_{\sigma} \rho(\mu) d \mu=\frac{2}{c} \text {. }
$$

Proof of Proposition 13: We prove first Eq. (2.50) for $V^{\prime}(a+c \cos 2 u)=\cos 2 k u$ with $k \geq 0$. $\mathbf{B}$ is symmetric over changing $\alpha \rightarrow-\alpha, \beta \rightarrow-\beta, \alpha \leftrightarrow \beta$. Hence, we can assume $\beta \geq \alpha \geq 0$. For odd $\alpha$ or $\beta$ we will have 0 , because of the ratio under the integral in Eq. (2.49) does not change after the shift $u \rightarrow \pi+u$ and $v \rightarrow \pi+v$. Therefore we change $\alpha \rightarrow 2 \alpha$ and $\beta \rightarrow 2 \beta$. And finally, changing $2 u \rightarrow u$ and $2 v \rightarrow v$ in Eq. (2.49), we obtain

$$
\mathbf{B}_{2 \alpha, 2 \beta}^{(k)}:=\frac{1}{4 \pi^{2} c} \int_{-\pi}^{\pi} d u \int_{-\pi}^{\pi} d v \frac{\cos k u-\cos k v}{\cos u-\cos v} \cos \alpha u \cos \beta v .
$$

To compute this integral we use two evident relations

$$
\int_{-\pi}^{\pi} \frac{\sin l u}{\sin u} d u=2 \pi \operatorname{sign} l \cdot \begin{cases}1, & 1 \text { is odd, } \\ 0, & 1 \text { is even. }\end{cases}
$$




$$
\int_{-\pi}^{\pi} \frac{\cos l u-\cos l v}{\cos u-\cos v} d v=2 \pi \frac{\sin l u}{\sin u} .
$$

Now to apply Eqs. (3.8) and (3.9) we transform integral for $\mathbf{B}_{2 \alpha, 2 \beta}^{(k)}$ as follows

$$
\begin{aligned}
\mathbf{B}_{2 \alpha, 2 \beta}^{(k)} & =\frac{1}{4 \pi^{2} c} \int_{-\pi}^{\pi} d u \int_{-\pi}^{\pi} d v(\cos k u-\cos k v) \frac{\cos \alpha u-\cos \alpha v}{\cos u-\cos v} \cos \beta v \\
& +\frac{1}{4 \pi^{2} c} \int_{-\pi}^{\pi} d u \int_{-\pi}^{\pi} d v \frac{\cos k u-\cos k v}{\cos u-\cos v} \cos \alpha v \cos \beta v
\end{aligned}
$$

We write the first integral as a sum of two parts: one with $\cos k u$ and the other with $\cos k v$. Then in the second part we integrate over $u$ using Eq. (3.9). In the second integral above we similarly integrate over $u$ and use Eq. (3.9). Then we obtain

$$
\begin{aligned}
\mathbf{B}_{2 \alpha, 2 \beta}^{(k)} & =\frac{1}{4 \pi^{2} c} \int_{-\pi}^{\pi} d u \cos k u \int_{-\pi}^{\pi} d v \frac{\cos \alpha u-\cos \alpha v}{\cos u-\cos v} \cos \beta v \\
& +\frac{1}{2 \pi c} \int_{-\pi}^{\pi} d v \frac{\sin k v-\sin \alpha v}{\sin v} \cos \alpha v \cos \beta v .
\end{aligned}
$$

Note that the function $f_{u}(v):=\frac{\cos \alpha u-\cos \alpha v}{\cos u-\cos v}$ can be represented as a trigonometric polynomial of $v$ with degree not bigger than $\alpha-1$, therefore the first integral in the above relation is equal to 0 if $\beta \geq \alpha$. And finally we have

$$
\begin{aligned}
\mathbf{B}_{2 \alpha, 2 \beta}^{(k)} & =\frac{1}{4 \pi c} \int_{-\pi}^{\pi} \frac{\sin (k-\alpha-\beta) v+\sin (k-\alpha+\beta) v}{\sin v} d v \\
& =\left\{\begin{array}{c}
c^{-1}, k-\alpha-\beta \in 2 \mathbb{N}+1 \\
0, \quad \begin{array}{c}
\text { otherwise }
\end{array}
\end{array}\right.
\end{aligned}
$$

Now using Eq. (3.10) and the Fourier expansion $V^{\prime}(a+c \cos 2 \phi)=\sum_{k=0}^{\infty} v_{2 k} \cos 2 k \phi$, we obtain the first equality in Eq. (2.50). To estimate $\mathbf{B}_{2 \alpha, 2 \beta}$ we use the Schwarz inequality

$$
\left|\sum_{k-\alpha-\beta \in 2 \mathbb{N}+1} v_{2 k}\right| \leq \sqrt{\sum_{k>2 \alpha+2 \beta} v_{k}^{2} k^{6}} \sqrt{\sum_{k>2 \alpha+2 \beta} \frac{1}{k^{6}}}=\underline{O}\left((\alpha+\beta)^{-5 / 2}\right) \text {. }
$$

Proof of Lemma 14 Using Eq. (2.52) in Eq. (2.51) we obtain

$$
\begin{aligned}
\frac{b}{c} & +\frac{a}{c} \widetilde{x}_{k+1}-\frac{1}{2}\left(\widetilde{x}_{k}+\widetilde{x}_{k+2}\right)+v_{0}\left(\frac{c}{2} \widetilde{x}_{k-1}+\frac{1+a}{2}\left(-\widetilde{x}_{k}+\widetilde{x}_{k+1}\right)-\frac{c}{2} \widetilde{x}_{k+2}\right) \\
& +\sum_{l=-\infty}^{\infty}\left[\frac{c^{2}}{4}\left(\mathbf{B}_{|4 l+2|}-\mathbf{B}_{|4 l-2|}\right)\left(\widetilde{x}_{k+2 l-1}-\widetilde{x}_{k+2 l+1}\right)\right. \\
& +\frac{c^{2}}{4}\left(\mathbf{B}_{|4 l+4|}-\mathbf{B}_{|2 l-2|+|2 l+2|}\right)\left(\widetilde{x}_{k+2 l+2}-\widetilde{x}_{k+2 l}\right) \\
& -\frac{b^{2}}{2}\left(\mathbf{B}_{|4 l-2|}-\mathbf{B}_{|4 l-4|}\right)\left(\widetilde{x}_{k+2 l-2}+\widetilde{x}_{k+2 l-1}\right) \\
& -\frac{b^{2}}{2}\left(\mathbf{B}_{|4 l+2|}-\mathbf{B}_{|2 l-2|+|2 l+2|}\right)\left(\widetilde{x}_{k+2 l}+\widetilde{x}_{k+2 l+1}\right) \\
& \left.+b^{2}\left(\mathbf{B}_{|4 l|}-\mathbf{B}_{|4 l-2|}\right)\left(\widetilde{x}_{k+2 l-1}+\widetilde{x}_{k+2 l}\right)\right]=\frac{b}{c}\left(1+\frac{k}{n}\right)-\frac{1}{c} \widetilde{x}_{k}+R_{k},
\end{aligned}
$$


where $R_{k}$ accumulates the errors of Eqs. (2.51) and (2.52). It is easy to see that

$$
\left|R_{k}\right| \leq C\left(\frac{k^{2}}{n^{2}}+S_{k}+\sum_{l=-\infty}^{\infty}\left(S_{k}+S_{k+2 l}\right)\left(\left|\mathbf{B}_{|4 l-4|}\right|+\ldots+\left|\mathbf{B}_{|4 l+4|}\right|\right)\right)+r_{k}^{(n)} .
$$

Recollecting terms with $\tilde{x}_{j}$ we obtain the system (3.11) in the form (2.53). Definitions (2.53) and (2.54) and system (3.11) imply

$$
\begin{gathered}
\delta(\phi)=A_{0}+2 \sum_{l=1}^{\infty} A_{l} \cos (l \phi) \\
=-a v_{0}+\frac{1-(1+a) v_{0}}{2}+c v_{0} \cos \phi+v_{2}\left(\frac{c}{4}-\frac{c}{2} \cos 2 \phi+2 a \cos \phi+2 a\right) \\
+\sum_{l=1} v_{4 l}\left(\frac{c}{2}(\cos (2 l+1) \phi-\cos (2 l-1) \phi)-2 a \cos 2 l \phi-2 a-4 a\left(\sum_{k=1}^{2 l-1} \cos k \phi\right)\right) \\
+\sum_{l=2} v_{4 l-2}\left(\frac{c}{2}(\cos (2 l-2) \phi-\cos (2 l) \phi)+2 a \cos (2 l-1) \phi+2 a+4 a\left(\sum_{k=1}^{2 l-2} \cos k \phi\right)\right) \\
=\left(c \sin \phi+2 a \cot \frac{\phi}{2}\right) \sum_{l=1}^{\infty}(-1)^{l-1} \sin l \phi v_{2 l}-\frac{c}{4} v_{2}+v_{0}\left(-a-\frac{1+a}{2}+c \cos \phi\right)+\frac{1}{2} \\
=-2\left(a+c \sin ^{2} \frac{\phi}{2}\right)\left(\sum_{l=1}^{\infty} v_{2 l}(-1)^{l} \frac{\sin l \phi}{\sin \phi} 2 \cos ^{2} \frac{\phi}{2}+v_{0}\right) .
\end{gathered}
$$

On the other hand, from the definition (1.10) we have

$$
P\left(2 \arcsin \left(\sin \frac{\theta}{2} \sin \frac{v}{2}\right)\right)=2 \pi \sqrt{2\left(1-c \sin ^{2} \frac{v}{2}\right)}\left((1-\cos v) \sum_{l=1}^{\infty} v_{2 l} \frac{\sin l v}{\sin v}-v_{0}\right) .
$$

The equality can be obtained from Eq. (1.10) by the change of variables $\sin \lambda / 2=\sin \theta / 2 \sin u / 2$ and $\sin \mu / 2=\sin \theta / 2 \sin v / 2$

$$
\begin{aligned}
P(\mu)= & \int_{-\theta}^{\theta} \frac{V^{\prime}(\lambda)-V^{\prime}(\mu)}{\sin \frac{\lambda-\mu}{2}} \frac{d \lambda}{\sqrt{\cos \lambda-\cos \theta}} \\
= & \sqrt{2} \int_{-\pi}^{\pi} \frac{\sin v / 2 \cos \mu / 2 V^{\prime}(a+c \cos v)-\sin u / 2 \cos \lambda / 2 V^{\prime}(a+c \cos u)}{\sin u / 2 \cos \mu / 2-\sin v / 2 \cos \lambda / 2} \frac{d u}{\cos \lambda / 2} \\
= & \sqrt{2} \int_{-\pi}^{\pi} \frac{\cos \mu / 2\left(\sin ^{2} v / 2 V^{\prime}(a+c \cos v)-\sin ^{2} u / 2 V^{\prime}(a+c \cos u)\right)}{\sin ^{2} u / 2-\sin ^{2} v / 2} d u \\
= & \sqrt{2} \cos \mu / 2\left((1-\cos v) \int_{-\pi}^{\pi} \frac{V^{\prime}(a+c \cos u)-V^{\prime}(a+c \cos v)}{\cos u-\cos v} d u-2 \pi v_{0} .\right)
\end{aligned}
$$

Now, using the definition of $v_{2 l}$ and equality

$$
\int_{-\pi}^{\pi} \frac{\cos l u-\cos l v}{\cos u-\cos v} d u=2 \pi \frac{\sin l v}{\sin v},
$$

we obtain Eq. (3.13). Therefore,

$$
\delta(\phi)=\frac{\sqrt{1-c \cos ^{2} \frac{\phi}{2}}}{\pi \sqrt{2}} P\left(-2 \arcsin \left(\sin \frac{\theta}{2} \cos \frac{\phi}{2}\right)\right) .
$$


To estimate the reminder we will use the inequality

$$
\left|\int_{-\infty}^{\infty} f(x) \overline{g(x)} d x\right| \leq \sqrt{\frac{1}{2 \pi \Gamma(2 s)} \int_{0}^{\infty} e^{-y} y^{2 s-1}\left\|f \star P_{y}\right\|_{2}^{2} d y} \sqrt{\int_{-\infty}^{\infty}|\widehat{g}(\xi)|^{2}(2|\xi|+1)^{2 s} d \xi},
$$

where $P_{y}(t)=\frac{y}{\pi\left(y^{2}+t^{2}\right)}, g(x)$ any $s$-times differentiable function with $g^{(s)} \in L_{2}$ and $f \in L_{2}$. The inequality was proposed in Ref. 6. It can be obtained from the Schwartz inequality

$$
\begin{aligned}
\left|\int_{-\infty}^{\infty} f(x) \overline{g(x)} d x\right| & =\left|\frac{1}{2 \pi} \int_{-\infty}^{\infty} \widehat{f}(\xi) \widehat{\widehat{g}(\xi)} d \xi\right| \\
\leq & \frac{1}{2 \pi}\left(\int_{-\infty}^{\infty}|\widehat{f}(\xi)|^{2}(2|\xi|+1)^{-2 s} d \xi\right)^{1 / 2}\left(\int_{-\infty}^{\infty}|\widehat{g}(\xi)|^{2}(2|\xi|+1)^{2 s} d \xi\right)^{1 / 2}
\end{aligned}
$$

and exact computation of the Fourier transform of $P_{y}$

$$
\widehat{P}_{y}(\xi)=\frac{1}{\pi} \int_{-\infty}^{\infty} \frac{y}{y^{2}+t^{2}} e^{-i t \xi} d t=\operatorname{sign}(y) e^{-|y||\xi|} .
$$

Now, using that

$$
\left\|f \star P_{y}\right\|_{2}^{2}=\frac{1}{2 \pi}\left\|\widehat{f} \widehat{P}_{y}\right\|_{2}^{2}
$$

and the definition of the $\Gamma$ function we obtain

$$
\frac{1}{\Gamma(2 s)} \int_{0}^{\infty} e^{-y} y^{2 s-1}\left|\widehat{P}_{y}(\xi)\right|^{2} d y=\frac{1}{\Gamma(2 s)} \int_{0}^{\infty} e^{-y(2|\xi|+1)} y^{2 s-1} d y=(2|\xi|+1)^{-2 s} .
$$

Finally, combining the above relations, we get Eq. (3.16). We use this inequality for $\widehat{V^{\prime}}$ and $\widehat{r}(\xi ; d)$ with $s=3$.

$$
\begin{aligned}
\left|\frac{1}{2 \pi} \int \widehat{V}^{\prime}(\xi) \widehat{r}(\xi ; d) d \xi\right|^{2} & \leq C_{V} \int_{0}^{\infty} e^{-y} y^{5}\left\|r(d) \star P_{y}\right\|_{2}^{2} d y \\
& =C_{V} \int_{0}^{\infty} e^{-y} y^{5}\left\|\left(f-f^{0}-f^{1}\right) \star P_{y}\right\|_{2}^{2} d y .
\end{aligned}
$$

On the other hand, for any self-adjoint linear operator $A$ and any linear $B$ we have

$$
\begin{gathered}
\frac{1}{2 \pi} \int e^{i x \xi} \widehat{P_{y}}(\xi) e^{i A \xi} d \xi=\frac{1}{\pi} \mathfrak{J} G_{A}(-x+i y), \\
\frac{1}{2 \pi} \int e^{i x \xi} \widehat{P}_{y}(\xi) e^{i A \xi t} B e^{i A \xi(1-t)} d \xi=\frac{1}{\pi} \mathfrak{J} G_{A}(-x+i y) B G_{A}(-x+i y),
\end{gathered}
$$

where $G_{A}(z)=(A-z)^{-1}$. Hence from Eqs. (2.43) and (3.17) we have

$$
\begin{gathered}
\left(f \star P_{y}\right)(x)=\frac{1}{\pi} \mathfrak{J} R_{k+d, k-1}(-x+i y), \\
\left(f^{0} \star P_{y}\right)(x)=\frac{1}{\pi} \mathfrak{J} R_{k+d, k-1}^{\star}(-x+i y), \\
\left(f^{1} \star P_{y}\right)(x)=\frac{1}{\pi} \mathfrak{J}\left\{R^{\star}(-x+i y) \delta M R^{\star}(-x+i y)\right\}_{k+d, k-1} .
\end{gathered}
$$

Using the resolvent identity, (3.18), (3.19), and (3.20) we obtain

$$
\begin{aligned}
\left(r \star P_{y}\right)(x)=\left(f \star P_{y}\right) & (x)-\left(f^{0} \star P_{y}\right)(x)-\left(f^{1} \star P_{y}\right)(x) \\
= & \mathfrak{J}\left\{R^{\star}(-x+i y) \delta M R(-x+i y) \delta M R^{\star}(-x+i y)\right\}_{k+d, k-1} .
\end{aligned}
$$


Now we are ready to estimate $r(d) \star P_{y}$.

$$
\begin{gathered}
\left|r(d) \star P_{y}\right| \leq\left|\left\langle R^{\star}(-x+i y) \delta M R(-x+i y) \delta M R^{\star}(-x+i y) e_{k-1}, e_{k+d}\right\rangle\right| \\
\leq\|R\| \sqrt{\left(R^{\star}(-x+i y) \delta M^{2} R^{\star}(-x-i y)\right)_{k+d, k+d}} \\
\cdot \sqrt{\left(R^{\star}(-x-i y) \delta M^{2} R^{\star}(-x+i y)\right)_{k-1, k-1}} .
\end{gathered}
$$

Both roots can be estimated using the definition of $\delta M$ and Eq. (2.59). Finally we obtain

$$
\left|r(d) \star P_{y}\right| \leq\|R\| \sum_{s=-\infty}^{\infty}\left|G_{2 s}\right|^{2} S_{k+2 s}
$$

For sufficiently small $y$ we will use another bound

$$
\begin{aligned}
&\left|r(d) \star P_{y}\right| \leq\left|\left(f \star P_{y}\right)(x)\right|+\left|\left(f^{0} \star P_{y}\right)(x)\right|+\left|\left(f^{1} \star P_{y}\right)(x)\right| \\
& \leq C\left(\|R\|+\left\|R^{\star}\right\|+\|\delta M\|\right.\left.\left(\left\{R^{\star}(z) R^{\star}(\bar{z})\right\}_{k+d, k+d}+\left\{R^{\star}(z) R^{\star}(\bar{z})\right\}_{k-1, k-1}\right)\right) \\
& \leq C\left(\|R\|+\left\|R^{\star}\right\|+\sum_{d=-2}^{d=2}\left\{R^{\star}(z) R^{\star}(\bar{z})\right\}_{k+d, k+d}\right) .
\end{aligned}
$$

Proof of Proposition 15 It is easy to see that we can prove this proposition for even $p$. For odd $p$ it follows from the Schwartz inequality. The definition (2.59) implies

$$
\frac{1}{a-z+c \cos 2 \phi}=\sum_{s} G_{2 s} e^{-2 i s \phi} \text {. }
$$

Hence

$$
\begin{aligned}
& \sum_{s}\left|G_{2 s}\right|^{2} s^{2 p}=\sum_{s} G_{2 s} s^{2 p} \frac{1}{2 \pi} \int_{-\pi}^{\pi} \frac{e^{-2 i s \phi}}{a-\bar{z}+c \cos 2 \phi} d \phi \\
&=\left(-\frac{1}{4}\right)^{p} \frac{1}{2 \pi} \int_{-\pi}^{\pi}\left(\frac{\partial^{2 p}}{\partial \phi^{2 p}} \frac{1}{a-z+c \cos 2 \phi}\right) \frac{1}{a-\bar{z}+c \cos 2 \phi} d \phi \\
&=C_{p} \int_{-\pi}^{\pi}\left|\frac{\partial^{p}}{\partial \phi^{p}} \frac{1}{a-z+c \cos 2 \phi}\right|^{2} d \phi \leq C_{p}^{\prime} y^{-2 p} \int_{-\pi}^{\pi} \frac{d \phi}{|a-z+c \cos 2 \phi|^{2}} d \phi \\
&=C_{p}^{\prime \prime} y^{-2 p-1} \mathfrak{J} G_{0} .
\end{aligned}
$$

From the residue theorem we obtain

$$
G_{0}=\frac{2 \zeta}{c\left(\zeta^{2}-1\right)}, \quad \text { where } \zeta^{2}+2 \frac{a-z}{c} \zeta+1=0 \text { and }|\zeta|<1
$$

Therefore,

$$
\left|G_{0}\right|=\frac{1}{\sqrt{|z-1||z-\cos \theta|}} .
$$

Finally, combining all above bounds, we obtain

$$
\begin{array}{r}
\int_{-\infty}^{\infty}\left(\sum_{s}\left|G_{2 s}\right|^{2} s^{2 p}\right)^{2} d x \leq C_{p}^{\prime \prime \prime} y^{-4 p-2} \int_{-\infty}^{\infty} \frac{d x}{|z-1||z-\cos \theta|} \\
\leq \widetilde{C}_{p} y^{-4 p-2} \max \{\log 1 / y, 1\}
\end{array}
$$




\section{ACKNOWLEDGMENTS}

The author is grateful to Professor M. Shcherbina for the problem statement and fruitful discussions. The author was partially supported by the Akhiezer fund.

${ }^{1}$ Albeverio, S., Pastur, L., and Shcherbina, M., "On the 1/n expansion for some unitary invariant ensembles of random matrices," Commun. Math. Phys. 224, 271-305 (2001).

${ }^{2}$ Baik, J., Deift, P., and Johansson, K., "On the distribution of the length of the longest increasing subsequence of random permutations," J. Am. Math. Soc. 12, 1119-1178 (1999).

${ }^{3}$ Cantero, M. J., Moral, L., and Velásquez, L., "Five-diagonal matrices and zeros of orthogonal polynomials on the unit circle," Linear Algebra and Its Applications 362, 29-56 (2003).

${ }^{4}$ Deift, P., Kriecherbauer, T., McLaughlin, K. T.-K., Venakides, S., and Zhou, X., "Uniform asymptotics for polynomials orthogonal with respect to varying exponential weights and applications to universality questions in random matrix theory," Commun. Pure Appl. Math. 52, 1335-1425 (1999).

${ }^{5}$ Hisakado, M., “Unitary matrix models and Painleve III,” Mod. Phys. Lett. A 11, 3001-3010 (1996).

${ }^{6}$ Johansson, K., "On fluctuations of eigenvalues of random Hermitian matrices,” Duke Math. J. 91, 151-204 (1998).

${ }^{7}$ Kolyandr, A., "On eigenvalue distribution of invariant ensembles of random matrices," Dop. Ukr. Ac. Sci.: mathematics 7, 14-20 (1997).

${ }^{8}$ Levin, E. and Lubinsky, D., "Universality limits in the bulk for varying measures," Adv. Math. 219(3), 743-779 (2008).

${ }^{9}$ McLaughlin, K. T.-R. and Miller, P. D., "The $\bar{\partial}$ steepest descent method and the asymptotic behavior of polynomials orthogonal on the unit circle with fixed and exponentially varying nonanalytic weights," Intern. Math. Res. Papers 48673, 1-77 (2006)

${ }^{10}$ McLaughlin, K. T.-R. and Miller, P. D., "The $\bar{\partial}$ steepest descent method for orthogonal polynomials on the real line with varying weights," Int. Math. Res. Notices 2008, 1-66 (2008).

${ }^{11}$ Mehta, M. L., Random Matrices (Academic, New York, 1991).

${ }^{12}$ Muskhelishvili, N. I., Singular Integral Equations (P. Noordhoff, Groningen, 1953).

${ }^{13}$ Pastur, L. and Shcherbina, M., "On the edge universality of the local eigenvalue statistics of matrix models," Mat. Fiz. Anal. Geom. 10(3), 335-365 (2003).

${ }^{14}$ Pastur, L. and Shcherbina, M., "Bulk Universality and related properties of Hermitian matrix model," J. Stat. Phys. 130, 205-250 (2007).

${ }^{15}$ Poplavskyi, M., "Bulk Universality for Unitary Matrix Models," Journal of Mathematical Physics, Analysis, Geometry 5(3), 245-274 (2009).

${ }^{16}$ Saff, E. B. and Totik, V., "Logarithmic potentials with external fields," Appendix B by Thomas Bloom, Grundlehren der Mathematischen Wissenschaften (Springer-Verlag, Berlin, 1997), Vol. 316

${ }^{17}$ Simon, B., Orthogonal polynomials on the unit circle. Part 1: Classical theory and Part 2: Spectral theory, AMS (Providence, 2005). 